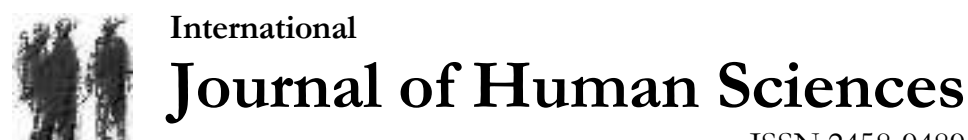 \\ ISSN:2458-9489
}

Volume 16 Issue 4 Year: 2019

\section{Comparison of activation rates in the main muscle group in the performance of Turkish folk dances ${ }^{1}$}

\author{
Kürşad Gülbeyaz ${ }^{2}$
}

\begin{abstract}
Folk dances are "spiritual culture" in terms of music, lyrics, movement and presentation features. Also, folk dances are the "material culture" in terms of instruments, clothes and equipment. Folk dances are intertwined with many disciplines, due to its different features such as music, instrument, language, religion, literature, history, clothes, sport, stage, art, sociology, consideration and intelligence.

There is no folk dance without music and rhythm. However, almost all of our women's dances and most of our men's dances are verbal. Therefore, folk dances are composed of lyrics, music and movement.

Folk dances have a sportive dimension as well as a cultural dimension. Although folk dances have been examined with the dimension of movement until present day, we see that the researches on physical, physiological and kinesiological aspects are not sufficient. The main purpose of this study was to determine the contraction rates of muscles during the performance of folk dances.

In this study, it has been identified 42 dances in Turkey, which movement and structural features are different from each other. These dances include 6 different "bar" type, 12 of "halay" type, 8 of "horon" type, 5 of "karşlama" type, and 11 of "zeybek" type. The study was completed using these dances.

The study is derived from the author's "Analysis of Turkish Folk Dances from the Perspective of Movement Science and Culture" doctoral dissertation titled.

The study was conducted in the main muscle group according to expert opinions and recommendations. The main muscle groups are Tibialis Anterior, Quadriceps Femoris, Flexor Digitorum Superficialis, Biceps Brachii, Longissimus Thoracis and Trapezius muscles.

Electrodes were attached to the main muscles used in the folk dances. The measurements were obtained by bio-trace programme and data were defined in microvolt.

As a result of the study, in the performance of Turkish folk dances on the type at the scale of Turkey, left Tibialis Anterior muscle (112.40), left Quadriceps Femoris muscle (112.32); the right Flexor Digitorum Superficialis muscle in the arm (49.63), the right Biceps Brachii muscle (60.07); the left Longissimus Thoracis muscle (46.68) and the right Trapezius muscle (104.19) contracted more.

Determining the ratio in muscle contractions in folk dances provides valuable information which can be used in coordination exercises, motor development, training in a specific sport and also in physical therapy.
\end{abstract}

Keywords: Turkish folk dances, Turkish folklore, sports, muscle, activation.

\footnotetext{
${ }^{1}$ The study is an extended version of the oral presentation presented at the 17 th Turkish World Social Sciences Congress held between 30 October - 01 November 2019.

2 Assoc. Dr. Muğla Sitkı Koçman University Faculty of Sport Sciences, kursadgulbeyaz@hotmail.com, kursadgulbevaz@mu.edu.tr
} 
Gülbeyaz, K. (2019). Comparison of activation rates in the main muscle group in the performance of Turkish folk dances. Journal of Human Sciences, 16(4), 1133-1144. doi:10.14687/jhs.v16i4.5888

\section{INTRODUCTION}

Cultural assets are divided into two groups as material and spiritual. We can group them abstract and concrete conceptually. The elements of material culture are concrete that means visible and touchable. The elements of spiritual culture are abstract and difficult to store. (Öter ve Ünal, 2017: 231). Folk dances; in terms of music, lyric, movement, expression, emotion transfer, are under the roof of our spiritual cultural values. Folk dances; considering the elements such as music instruments, clothes, jewelry and accessories, tools and equipment, it can be examined within the scope of our material cultural values. Folklore is a science that includes all cultural products in the material and spiritual fields belonging to the people living in a specific geographical area. Folklore aims to investigate, compile, classify, interpret and conclude these cultural products in their own way. (Örnek, 2000: 15). Folk dances, although considered as a sub-branch of folklore, have an interdisciplinary structure. Folk dances are intertwined with many disciplines, due to its different features such as music, instrument, language, religion, literature, history, clothes, sport, stage, art, sociology, consideration and intelligence. Because of this feature, it would not be wrong to say; folk dance is an "interdisciplinary science".

As a concept, folk dance is music integration, which is supported by anonymous folk music consisting of sound units, which creates an aesthetic effect and excitement by means of measured and balanced movements arranged in a way that is pleasing to the eye and ear (Öngel, 1992: 8; Ekmekçioğlu, Bekar ve Kaplan, 2001: 18). Folk dances consist of movements. Movements, on the other hand, contain anything such as lyrics, emotions and thoughts, from the time without lyrics and language to the present. According to Huizinga (1955), dance precedes culture. Dance is not the result of the emergence of various cultures or coincidental, on the contrary, it was the main factor in the birth of various forms of culture. The origin of language and ritual is dance. Dance is an optional and voluntary act and is not bespoke or coerced.

Folk music and folk dance actually constitute two separate fields. On the other hand, the fields of usage are close. Music and dance are exhibited together, especially in the making of folk dances. Therefore, both should be considered together (Altuğ, 1988: 1). There is no folk dance without music or rhythm. However, there is lyric more than half of the folk dances, although not used much today. Especially almost all of our women's dances and most of our men's dances contain lyrics. Therefore, folk dances are composed of "lyric, music and movement". Traditional clothing is another inseparable part of our dances. Traditional clothes contain the knowledge that the Turkish nation has gained since its existence. Folk dance is one of the most important elements of Turkish culture and Turkish folklore as it includes many material and spiritual elements such as lyrics, music, movement, clothes, emotion transfer.

In addition, folk dances have a cultural dimension as well as a sportive dimension. Folk dance is a cultural product of art and sport value, which is composed of symbolic ordering of harmonious movements by integrating with rhythm and melody, resulting from the reflections of people's relations with each other (human-human) and with nature (human-nature). On the other hand, the formation of rhythmic movements creates the sportive side (Aydın, 1982: 5).

While performing folk dances, there are some changes in the metabolism, especially in the respiratory and circulatory systems. This shows us that folk dance is an important physical activity (Kaya, 2016: 11-12). Regular exercise is considered as the basic element of healthy life. One of the most important goals of physical activities is to keep at the appropriate level of the person physical appropriacy and conditional level. Considering the fact that people of all ages can perform folk dances, it can be used to give people the habit of physical activity and regular exercise. Therefore, the sportive characteristics of folk dances should be examined. (Gülbeyaz, 2018: 75). Even though dance has been studied with the dimension of movement, researches are lacking especially in terms of physical, physiological and 
kinesiological aspects. In the light of all these, the importance of quantitative researches, considering the sporting characteristics of folk dances, is seen.

\section{PURPOSE}

Folk dance is a set of regular and rhythmic movements. It also has a sportive dimension in this respect. Even though folk dances have been studied with the dimension of movement, researches are lacking especially in terms of physical, physiological and kinesiological aspects. The aim of this study is to determine the contraction rates of the main muscles used while dancing.

\section{RESTRICTION}

Turkish folk dances are classified under 5 main types: bar, halay, horon, karşılama and zeybek. In terms of diversity, our folk dances are almost numerous. When we examine our dances on the provincial or regional basis, they are all very close to each other in terms of structural and movements. Therefore, dances which have different structural and motional characteristics were used in this study. In this study, it has been identified 42 dances in Turkey, which movement and structural features are different from each other. These dances include 6 different "bar" type, 12 of "halay" type, 8 of "horon" type, 5 of "karşlama" type, and 11 of "zeybek" type.

The dances were performed by two male dancers. Based on expert opinions, these two dancers' lifestyle, body and muscle structures were similar to each other.

\section{METHOD}

EMG / ENMG (Electroneuromyography) technique was used in this study. EMG refers to the printing of electrical signals from nerves and muscles. In this study, the main muscle group muscles were used by taking the opinions of experts in the area. Main muscle group; Tibialis Anterior and Quadriceps Femoris muscles in the legs, Flexor Digitorum Superficialis and Biceps Brachii muscles in the arms and Longissimus Thoracis and Trapezius muscles in the back.

Two electrodes were attached to these muscles and the selected dances were performed. Each dance was performed three times. The muscles of the leg in the first dance, the arm muscles in the second dance and the back muscles in the third dance were measured. The minimum, maximum, average and standard deviation values of the measurement results were obtained by bio-trace programme. These data were defined in microvolt.

The average value of the data obtained on the basis of the dance to the rate of activation of some of the major muscle has reached the ratio of arithmetic mean taking kind of the ratio of the activation of some of the main muscle and the ratio of the arithmetic mean by taking the ratio of the activation of the main muscles in the scale of Turkey.

\section{WHICH MUSCLES WERE MEASURED IN THE STUDY?}

\section{Tibialis Anterior Muscle}

This muscle makes dorsiflexion and supination movements to the foot and supports the foot arch. Together with the peroneus longus muscle forms the stirrup. When the foot is immobilized, it brings the leg closer to the foot. Especially it is used for speed walking and skiing (Premkumar, 2015: 257; Weineck, 2011: 121). 


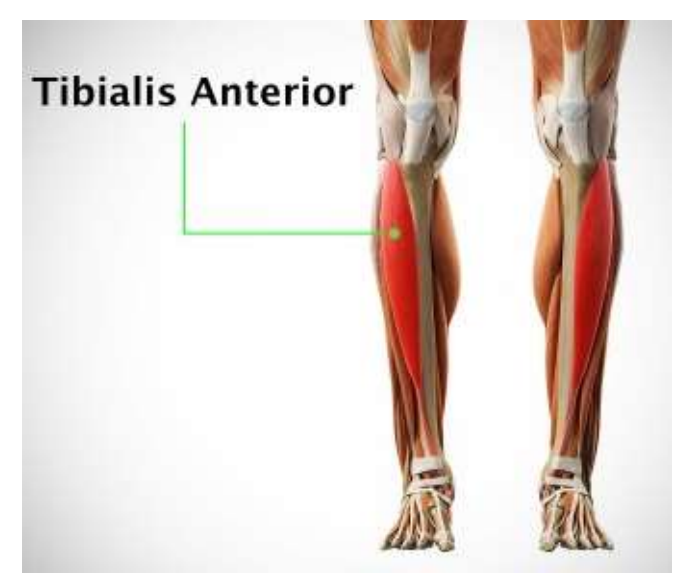

Picture 1: Tibialis Anterior Muscle

\section{Quadriceps Femoris Muscle:}

The most important extensor of the knee joint, the quadriceps femoris muscle is the largest and strongest muscle in human. It prevents knee bending while standing. In addition, as in all running and jumping exercises, it provides the strong extension of knee. Also, the rectus femoris muscle makes the flexion movement to hip. (Premkumar, 2015: 250-251; Weineck, 2011: 89-90).

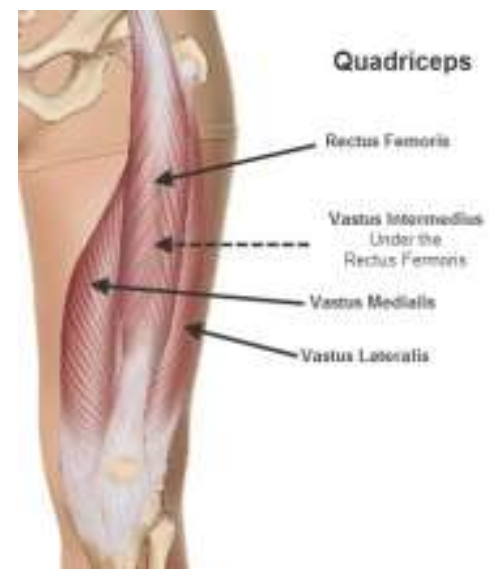

Picture 2: Quadriceps Femoris Muscle

\section{Flexör Digitorum Superficialis Muscle}

It's a two-headed muscle. One of them flexor digitorium superficialis muscle attaches to the body of Radius. The other is the humero ulnar head. In the distal, the flexor passes under the retineculum and attaches to the phalanx stems of 1-2-3-4 fingers. It helps in volar flexion movement of fingers, flexion movement of wrist joint and flexion movement of elbow. It performs synergist function in supination (Kaya, 2004: 102). 


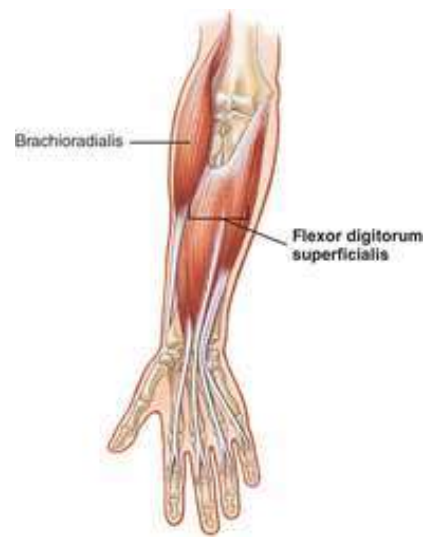

Picture 3: Flexör Digitorum Superficialis Muscle

\section{Biceps Brachii Muscle}

It helps flexion and supination movements of the forearm and abduction movement while the humerus is in external rotation. The long head of the muscle lifts the arm from the attached position and rotates inward. The short head makes adduction. It participates in the swinging movement of the arms during walking. The long beam was made to stabilize the shoulder joint by originating in the upper labrum of the glenoid fossa (Premkumar, 2015: 220; Weineck, 2011: 79).

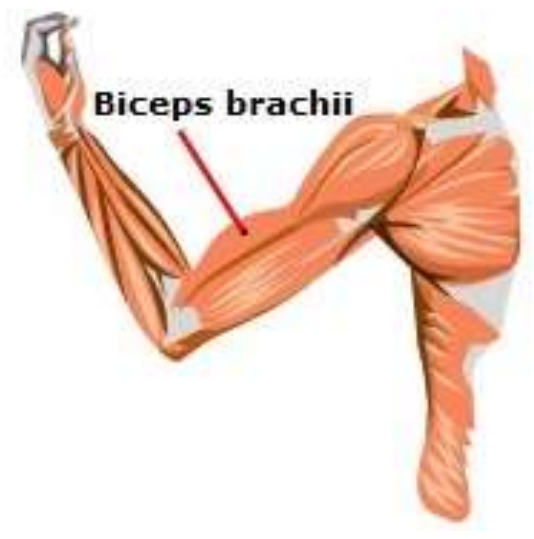

Picture 4: Biceps Brachii Muscle

\section{Longissimus Thoracis Muscle}

It extends the spine and bends the spine to the same side. Unidirectional stimulation results in lateral flexion of some segments of the spine (Premkumar, 2015: 190).

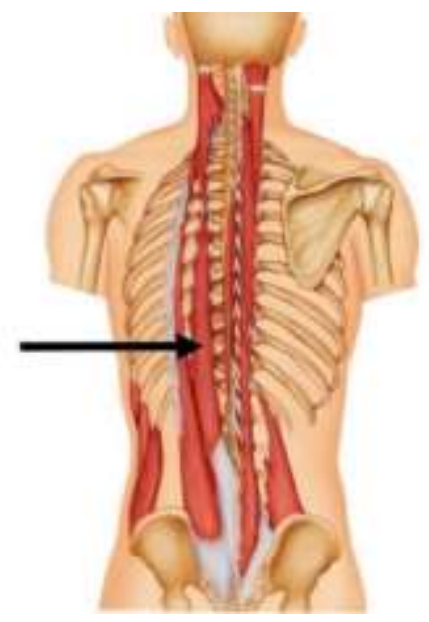

Picture 5: Longissimus Thoracis Muscle 


\section{Trapezius Muscle}

It helps to move the shoulder backwards, upwards and downwards. It is innervated by cervical nerve (Kaya, 2004: 102).

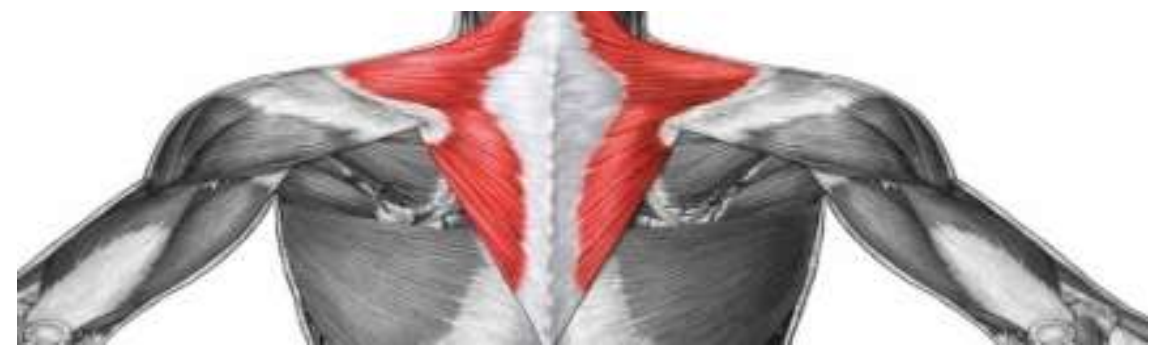

Picture 6: Trapezius Muscle

\section{RESULTS}

Table 1. Activation Rates of Main Muscles in Performing to Bar Type Dances

\begin{tabular}{|c|c|c|c|c|c|c|c|c|}
\hline & 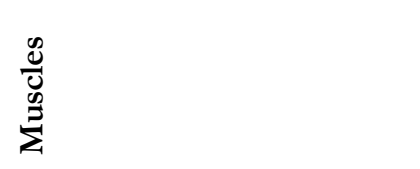 & 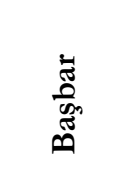 & 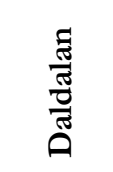 & 竞 & 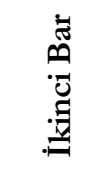 & 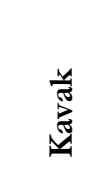 & 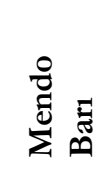 & $\frac{\widetilde{a}}{\tilde{\Xi}}$ \\
\hline \multirow{2}{*}{$\begin{array}{c}\text { Right } \\
\text { Leg }\end{array}$} & Tibialis Anterior & 116.08 & 126.80 & 100.17 & 168.48 & 102.69 & 93.49 & 117.95 \\
\hline & Quadriceps Femoris & 130.01 & 156.14 & 169.46 & 161.95 & 120.43 & 93.06 & 138.51 \\
\hline \multirow{2}{*}{$\begin{array}{l}\text { Left } \\
\text { Leg }\end{array}$} & Tibialis Anterior & 131.78 & 123.20 & 147.96 & 189.86 & 78.67 & 85.80 & 126.21 \\
\hline & Quadriceps Femoris & 102.95 & 114.20 & 127.64 & 152.68 & 62.65 & 138.26 & 116.40 \\
\hline \multirow{2}{*}{$\begin{array}{l}\text { Right } \\
\text { Arm }\end{array}$} & Flexör Digitorum Superficialis & 26.48 & 51.68 & 36.76 & 18.25 & 65.45 & 30.27 & 38.15 \\
\hline & Biceps Brachii & 57.00 & 74.84 & 37.31 & 44.27 & 67.22 & 45.58 & 54.37 \\
\hline \multirow{2}{*}{$\begin{array}{l}\text { Left } \\
\text { Arm }\end{array}$} & Flexör Digitorum Superficialis & 35.20 & 28.07 & 29.88 & 32.40 & 42.54 & 36.38 & 34.08 \\
\hline & Biceps Brachii & 75.93 & 84.37 & 33.17 & 61.51 & 74.61 & 59.14 & 64.79 \\
\hline \multirow{2}{*}{$\begin{array}{c}\text { Right } \\
\text { Bag }\end{array}$} & Longissimus Thoracis & 47.25 & 40.36 & 41.17 & 45.54 & 34.38 & 33.50 & 40.37 \\
\hline & Trapezius & 112.33 & 160.05 & 44.15 & 85.78 & 109.98 & 61.03 & 95.55 \\
\hline \multirow{2}{*}{$\begin{array}{l}\text { Left } \\
\text { Bag }\end{array}$} & Longissimus Thoracis & 38.14 & 38.87 & 31.36 & 35.47 & 28.11 & 62.15 & 39.02 \\
\hline & Trapezius & 155.39 & 180.33 & 43.13 & 105.43 & 146.56 & 59.28 & 115.02 \\
\hline
\end{tabular}

In the performance of "Bar" type dances, the left Tibialis Anterior muscle (126.21), right Quadriceps Femoris muscle (138.51) in the leg; the right Flexor Digitorum Superficialis muscle (38.15), the left Biceps Brachii muscle (64.79) in the arm; right Longissimus Thoracis muscle (40.37), left Trapezius muscle (105.02) on the back were contracted more. 
Table 2. Activation Rates of Main Muscles in Performing to Halay Type Dances

\begin{tabular}{|c|c|c|c|c|c|c|c|c|c|c|c|c|c|c|}
\hline & $\sum_{\sum}^{\stackrel{\infty}{0}}$ & 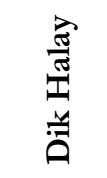 & $\frac{\vec{j}}{\tilde{I}}$ & 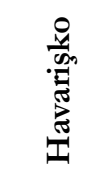 & 咅 & 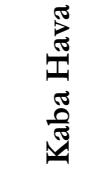 & $\underset{\pi}{*}$ & 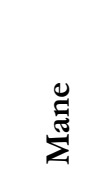 & 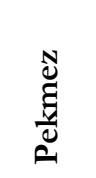 & $\begin{array}{l}\tilde{E} \\
\text { है } \\
\text { की }\end{array}$ & 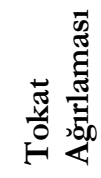 & $\begin{array}{l}\mathscr{0} \\
= \\
F\end{array}$ & 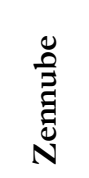 & $\frac{\mathscr{3}}{\tilde{J}}$ \\
\hline \multirow{2}{*}{$\begin{array}{c}\text { Right } \\
\text { Leg }\end{array}$} & $\begin{array}{l}\text { Tibialis } \\
\text { Anterior }\end{array}$ & 109.15 & 116.16 & 112.72 & 77.95 & 58.20 & 98.20 & 110.88 & 59.87 & 85.61 & 65.17 & 80.40 & 69.29 & 86.97 \\
\hline & $\begin{array}{l}\text { Quadriceps } \\
\text { Femoris }\end{array}$ & 90.15 & 148.87 & 86.94 & 107.98 & 29.35 & 118.10 & 82.93 & 49.80 & 85.76 & 60.92 & 66.90 & 64.13 & 82,65 \\
\hline \multirow{2}{*}{$\begin{array}{l}\text { Left } \\
\text { Leg }\end{array}$} & $\begin{array}{l}\text { Tibialis } \\
\text { Anterior }\end{array}$ & 85.88 & 133.84 & 98.88 & 96.85 & 65.78 & 117.61 & 85.27 & 28.51 & 65.15 & 75.63 & 97.33 & 44.02 & 82.90 \\
\hline & $\begin{array}{l}\text { Quadriceps } \\
\text { Femoris }\end{array}$ & 120.98 & 106.53 & 124.86 & 91.59 & 36.91 & 88.25 & 96.76 & 18.33 & 104.53 & 42.14 & 74.76 & 55.05 & 80.06 \\
\hline \multirow{2}{*}{$\begin{array}{l}\text { Right } \\
\text { Arm }\end{array}$} & $\begin{array}{l}\text { Flexör } \\
\text { Digitorum } \\
\text { Superficialis }\end{array}$ & 20.82 & 24.07 & 63.62 & 58.10 & 22.25 & 75.69 & 23.72 & 11.96 & 39.41 & 27.27 & 21.99 & 66.02 & 37.91 \\
\hline & $\begin{array}{l}\text { Biceps } \\
\text { Brachii }\end{array}$ & 21.15 & 8.40 & 85.93 & 41.69 & 57.63 & 62.56 & 22.57 & 25.67 & 51.67 & 36.11 & 49.48 & 71.04 & 44.49 \\
\hline \multirow{2}{*}{$\begin{array}{l}\text { Left } \\
\text { Arm }\end{array}$} & $\begin{array}{l}\text { Flexör } \\
\text { Digitorum } \\
\text { Superficialis }\end{array}$ & 34.37 & 18.91 & 50.91 & 38.04 & 30.47 & 34.69 & 32.55 & 12.05 & 36.28 & 18.42 & 31.55 & 41.84 & 31.67 \\
\hline & $\begin{array}{l}\text { Biceps } \\
\text { Brachii }\end{array}$ & 17.33 & 9.96 & 64.70 & 30.99 & 28.51 & 60.60 & 22.62 & 30.67 & 31.92 & 43.52 & 13.69 & 38.04 & 32.71 \\
\hline \multirow{2}{*}{$\begin{array}{c}\text { Right } \\
\text { Bag }\end{array}$} & $\begin{array}{l}\text { Longissimus } \\
\text { Thoracis }\end{array}$ & 38.34 & 42.73 & 51.17 & 42.06 & 20.69 & 40.82 & 30.66 & 25.45 & 32.43 & 30.29 & 32.00 & 27.15 & 34.48 \\
\hline & Trapezius & 68.84 & 46.12 & 85.09 & 64.23 & 120.20 & 57.29 & 73.95 & 55.47 & 95.01 & 77.79 & 59.34 & 94.42 & 74.81 \\
\hline \multirow{2}{*}{$\begin{array}{l}\text { Left } \\
\text { Bag }\end{array}$} & $\begin{array}{l}\text { Longissimus } \\
\text { Thoracis }\end{array}$ & 69.25 & 28.27 & 51.71 & 49.48 & 24.98 & 43.98 & 41.34 & 24.04 & 38.14 & 42.98 & 68.07 & 39.78 & 43.50 \\
\hline & Trapezius & 40.65 & 47.45 & 55.15 & 77.14 & 61.73 & 53.42 & 33.56 & 46.17 & 42.60 & 79.52 & 30.53 & 49.02 & 51.41 \\
\hline
\end{tabular}

In the performance of "Halay" type dances, the right Tibialis Anterior muscle (86.97) and the right Quadriceps Femoris muscle (82.65) in the leg; right Flexor Digitorum Superficialis muscle (37.91), right Biceps Brachii muscle (44.49) in the arm, left Longissimus Thoracis muscle (43.50), right Trapezius muscle (74.81) on the back were contracted more.

Table 3. Activation Rates of Main Muscles in Performing Horon Type Dances

\begin{tabular}{|c|c|c|c|c|c|c|c|c|c|c|}
\hline & $\frac{\mathscr{O}}{0}$ & 큼 & 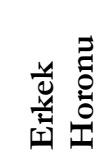 & 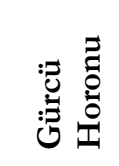 & 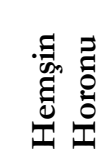 & 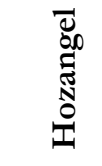 & Nㅗㄹ & 胥 & 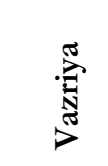 & $\frac{\widetilde{J}}{\tilde{\Xi}}$ \\
\hline \multirow{2}{*}{$\begin{array}{c}\text { Right } \\
\text { Leg }\end{array}$} & Tibialis Anterior & 144.91 & 104.34 & 166.22 & 127.86 & 112.73 & 97.45 & 128.01 & 103.31 & 123.10 \\
\hline & Quadriceps Femoris & 145.12 & 83.06 & 170.23 & 134.19 & 166.81 & 102.24 & 161.25 & 85.51 & 131.05 \\
\hline \multirow{2}{*}{$\begin{array}{l}\text { Left } \\
\text { Leg }\end{array}$} & Tibialis Anterior & 127.34 & 83.25 & 120.04 & 139.48 & 145.91 & 126.85 & 230.35 & 112.16 & 135.67 \\
\hline & Quadriceps Femoris & 220.45 & 93.56 & 124.55 & 190.04 & 113.31 & 99.61 & 191.06 & 143.91 & 147.06 \\
\hline \multirow{2}{*}{$\begin{array}{c}\text { Right } \\
\text { Arm }\end{array}$} & $\begin{array}{l}\text { Flexör Digitorum } \\
\text { Superficialis }\end{array}$ & 113.04 & 46.99 & 41.64 & 149.41 & 35.84 & 43.25 & 61.60 & 105.61 & 74.67 \\
\hline & Biceps Brachii & 158.49 & 48.56 & 61.99 & 170.04 & 52.01 & 66.24 & 81.49 & 121.87 & 95.09 \\
\hline $\begin{array}{l}\text { Left } \\
\text { Arm }\end{array}$ & $\begin{array}{l}\text { Flexör Digitorum } \\
\text { Superficialis }\end{array}$ & 136.28 & 47.53 & 36.24 & 169.29 & 34.00 & 42.81 & 42.13 & 75.90 & 73.02 \\
\hline
\end{tabular}


Gülbeyaz, K. (2019). Comparison of activation rates in the main muscle group in the performance of Turkish folk dances. Journal of Human Sciences, 16(4), 1133-1144. doi:10.14687/jhs.v16i4.5888

\begin{tabular}{clcccccccccc}
\hline & Biceps Brachii & 111.86 & 28.16 & 38.02 & 136.57 & 39.53 & 64.72 & 44.23 & 101.92 & 70.63 \\
$\begin{array}{l}\text { Right } \\
\text { Bag }\end{array}$ & $\begin{array}{l}\text { Longissimus } \\
\text { Thoracis }\end{array}$ & 73.31 & 49.30 & 38.58 & 79.37 & 49.57 & 46.89 & 87.10 & 62.49 & 60.83 \\
& $\begin{array}{l}\text { Trapezius } \\
\text { Left }\end{array}$ & $\begin{array}{l}\text { Longissimus } \\
\text { Bag }\end{array}$ & 122.25 & 104.37 & 68.08 & 180.68 & 97.01 & 73.97 & 96.70 & 123.42 & 108.31 \\
& Thoracis & 85.67 & 54.54 & 65.41 & 143.38 & 40.95 & 32.40 & 65.07 & 74.58 & 70.25 \\
\hline
\end{tabular}

In the performance of "Horon" type dances, the left Tibialis Anterior muscle (135.67) and the left Quadriceps Femoris muscle (147.06) in the leg; the right Flexor Digitorum Superficialis muscle (74.67), the right Biceps Brachii muscle (95.09) in the arm; the right Longissimus Thoracis muscle (60.83) and right Trapezius muscle (108.31) on the back were contracted more.

Table 4. Activation Rates of Main Muscles in Performing Karş1lama Type Dances

\begin{tabular}{|c|c|c|c|c|c|c|c|}
\hline & $\begin{array}{l}\frac{8}{0} \\
\frac{0}{0} \\
\sum^{0}\end{array}$ & $\underset{⿱ 乛}{\mathbb{E}}$ & 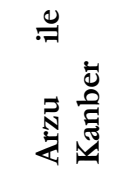 & 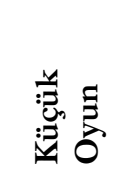 & $\begin{array}{l}\stackrel{0}{\infty} \\
\stackrel{e}{e} \\
\sum_{i}^{e}\end{array}$ & 胥 & 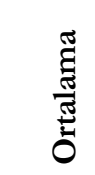 \\
\hline \multirow{2}{*}{$\begin{array}{l}\text { Right } \\
\text { Leg }\end{array}$} & Tibialis Anterior & 111.31 & 114.87 & 117.27 & 112.83 & 69.54 & 103.16 \\
\hline & Quadriceps Femoris & 129.18 & 115.94 & 130.98 & 111.26 & 72.96 & 112.06 \\
\hline \multirow{2}{*}{$\begin{array}{l}\text { Left } \\
\text { Leg }\end{array}$} & Tibialis Anterior & 114.98 & 127.36 & 104.47 & 128.58 & 102.45 & 115.57 \\
\hline & Quadriceps Femoris & 138.56 & 148.21 & 104.86 & 128.80 & 84.86 & 121.06 \\
\hline \multirow{2}{*}{$\begin{array}{l}\text { Right } \\
\text { Arm }\end{array}$} & Flexör Digitorum Superficialis & 41.33 & 66.96 & 53.67 & 78.70 & 19.83 & 52.10 \\
\hline & Biceps Brachii & 121.85 & 70.00 & 75.14 & 104.26 & 51.01 & 84.45 \\
\hline \multirow{2}{*}{$\begin{array}{l}\text { Left } \\
\text { Arm }\end{array}$} & Flexör Digitorum Superficialis & 41.38 & 47.64 & 34.41 & 91.98 & 20.86 & 47.25 \\
\hline & Biceps Brachii & 37.88 & 81.92 & 96.88 & 125.51 & 62.39 & 80.92 \\
\hline \multirow{2}{*}{$\begin{array}{c}\text { Right } \\
\text { Bag }\end{array}$} & Longissimus Thoracis & 63.48 & 40.12 & 38.10 & 44.42 & 31.17 & 43.46 \\
\hline & Trapezius & 92.85 & 158.60 & 114.39 & 117.91 & 103.14 & 117.38 \\
\hline \multirow{2}{*}{$\begin{array}{l}\text { Left } \\
\text { Bag }\end{array}$} & Longissimus Thoracis & 55.90 & 38.99 & 29.15 & 38.65 & 20.74 & 36.69 \\
\hline & Trapezius & 48.64 & 200.88 & 139.39 & 149.61 & 113.75 & 130.45 \\
\hline
\end{tabular}

In the performance of "karşılama" dances, the right Tibialis Anterior muscle (103.16) and the left Quadriceps Femoris muscle (121.06) in the leg; the right Flexor Digitorum Superficialis muscle (52.10), the right Biceps Brachii muscle (84.45) in the arm; the right Longissimus Thoracis muscle (43.46), the left Trapezius muscle (130.45) on the back were contracted more. 
Table 5. Activation Rates of Main Muscles in Performing Zeybek Type Dances

\begin{tabular}{|c|c|c|c|c|c|c|c|c|c|c|c|c|c|}
\hline & 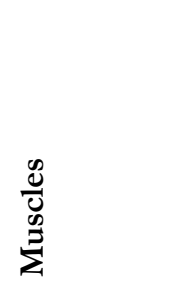 & 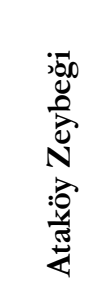 & 胥 & 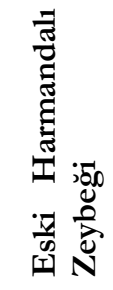 & 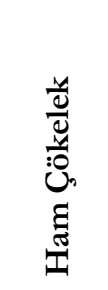 & 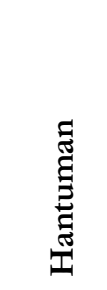 & 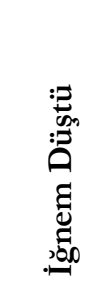 & 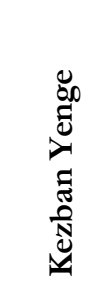 & 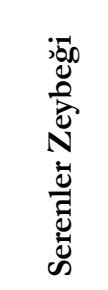 & 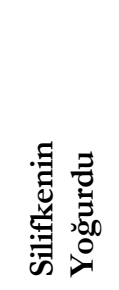 & 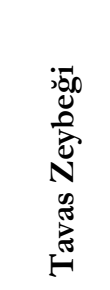 & : & 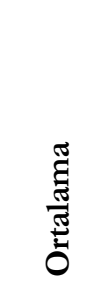 \\
\hline \multirow{2}{*}{$\begin{array}{l}\text { Right } \\
\text { Leg }\end{array}$} & $\begin{array}{l}\text { Tibialis } \\
\text { Anterior }\end{array}$ & 78.72 & 93.20 & 87.71 & 91.81 & 123.54 & 83.47 & 69.60 & 129.51 & 81.30 & 104.67 & 114.21 & 96.16 \\
\hline & $\begin{array}{l}\text { Quadriceps } \\
\text { Femoris }\end{array}$ & 58.77 & 95.16 & 58.09 & 79.42 & 101.34 & 68.94 & 60.85 & 74.80 & 69.05 & 98.45 & 139.41 & 82.21 \\
\hline \multirow{2}{*}{$\begin{array}{l}\text { Left } \\
\text { Leg }\end{array}$} & $\begin{array}{l}\text { Tibialis } \\
\text { Anterior }\end{array}$ & 84.54 & 103.31 & 78.87 & 86.13 & 101.17 & 100.07 & 99.52 & 120.20 & 101.90 & 116.37 & 125.63 & 101.61 \\
\hline & $\begin{array}{l}\text { Quadriceps } \\
\text { Femoris }\end{array}$ & 82.08 & 102.45 & 82.79 & 99.50 & 113.27 & 79.03 & 91.30 & 103.72 & 95.69 & 99.72 & 117.52 & 97.01 \\
\hline \multirow{2}{*}{$\begin{array}{l}\text { Right } \\
\text { Arm }\end{array}$} & $\begin{array}{l}\text { Flexör } \\
\text { Digitorum } \\
\text { Superficialis }\end{array}$ & 31.71 & 62.26 & 31.35 & 39.16 & 28.90 & 50.58 & 58.73 & 60.39 & 49.77 & 29.08 & 56.56 & 45.32 \\
\hline & $\begin{array}{l}\text { Biceps } \\
\text { Brachii } \\
\text { Flexör }\end{array}$ & 51.53 & 87.19 & 62.63 & 59.96 & 71.80 & 80.51 & 105.36 & 88.10 & 81.01 & 62.60 & 70.98 & 74.70 \\
\hline \multirow{2}{*}{$\begin{array}{l}\text { Left } \\
\text { Arm }\end{array}$} & $\begin{array}{l}\text { Digitorum } \\
\text { Superficialis }\end{array}$ & 32.86 & 74.59 & 48.80 & 30.76 & 43.55 & 44.37 & 66.14 & 59.87 & 52.93 & 43.10 & 47.91 & 49.53 \\
\hline & $\begin{array}{l}\text { Biceps } \\
\text { Brachii }\end{array}$ & 24.34 & 50.24 & 27.68 & 39.60 & 31.23 & 58.14 & 96.87 & 58.04 & 38.72 & 31.12 & 108.55 & 51.32 \\
\hline \multirow{2}{*}{$\begin{array}{l}\text { Right } \\
\text { Bag }\end{array}$} & $\begin{array}{l}\text { Longissimus } \\
\text { Thoracis }\end{array}$ & 29.35 & 46.61 & 28.07 & 32.71 & 38.56 & 29.30 & 36.26 & 33.17 & 36.00 & 30.58 & 49.14 & 35.43 \\
\hline & Trapezius & 132.10 & 130.02 & 117.12 & 103.81 & 177.85 & 102.29 & 79.12 & 124.91 & 129.03 & 167.01 & 110.50 & 124.89 \\
\hline \multirow{2}{*}{$\begin{array}{l}\text { Left } \\
\text { Bag }\end{array}$} & $\begin{array}{l}\text { Longissimus } \\
\text { Thoracis }\end{array}$ & 37.24 & 63.85 & 30.09 & 44.88 & 47.43 & 32.16 & 54.48 & 36.63 & 61.49 & 36.87 & 38.08 & 43.93 \\
\hline & Trapezius & 69.57 & 78.77 & 68.47 & 58.43 & 87.40 & 65.78 & 46.23 & 70.23 & 67.95 & 78.34 & 138.24 & 75.40 \\
\hline
\end{tabular}

In the performance of "zeybek" dances, left Tibialis Anterior Muscle (101.61), left Quadriceps Femoris Muscle (97.01) in the leg; the left Flexor Digitorum Superficialis muscle (49.53), the right Biceps Brachii muscle (74.70) in the arm; the left Longissimus Thoracis muscle (43.93) and the right Trapezius muscle (124.89) on the back were contracted more.

Table 6. Activation Rates of Main Muscles in Performing to Turkish Folk Dances by Type

\begin{tabular}{|c|c|c|c|c|c|c|c|}
\hline & 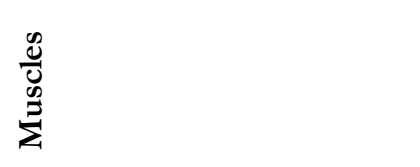 & ติ & 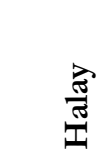 & 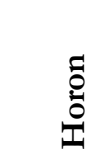 & 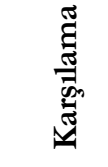 & $\frac{\sqrt[y]{d}}{\frac{0}{d}}$ & 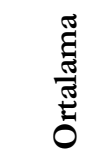 \\
\hline \multirow{2}{*}{$\begin{array}{c}\text { Right } \\
\text { Leg }\end{array}$} & Tibialis Anterior & 117.95 & 86.97 & 123.10 & 103.16 & 96.16 & 105.47 \\
\hline & Quadriceps Femoris & 138.51 & 82,65 & 131.05 & 112.06 & 82.21 & 109.30 \\
\hline \multirow{2}{*}{$\begin{array}{l}\text { Left } \\
\text { Leg }\end{array}$} & Tibialis Anterior & 126.21 & 82.90 & 135.67 & 115.57 & 101.61 & 112.40 \\
\hline & Quadriceps Femoris & 116.40 & 80.06 & 147.06 & 121.06 & 97.01 & 112.32 \\
\hline \multirow{2}{*}{$\begin{array}{l}\text { Right } \\
\text { Arm }\end{array}$} & Flexör Digitorum Superficialis & 38.15 & 37.91 & 74.67 & 52.10 & 45.32 & 49.63 \\
\hline & Biceps Brachii & 54.37 & 44.49 & 95.09 & 84.45 & 74.70 & 70.62 \\
\hline
\end{tabular}


Gülbeyaz, K. (2019). Comparison of activation rates in the main muscle group in the performance of Turkish folk dances. Journal of Human Sciences, 16(4), 1133-1144. doi:10.14687/ihs.v16i4.5888

\begin{tabular}{clcccccc}
\hline $\begin{array}{c}\text { Left } \\
\text { Arm }\end{array}$ & Flexör Digitorum Superficialis & 34.08 & 31.67 & 73.02 & 47.25 & 49.53 & 47.11 \\
& Biceps Brachii & 64.79 & 32.71 & 70.63 & 80.92 & 51.32 & 60.07 \\
$\begin{array}{c}\text { Right } \\
\text { Bag }\end{array}$ & Longissimus Thoracis & 40.37 & 34.48 & 60.83 & 43.46 & 35.43 & 42.92 \\
& Trapezius & 95.55 & 74.81 & 108.31 & 117.38 & 124.89 & 104.19 \\
$\begin{array}{c}\text { Left } \\
\text { Bag }\end{array}$ & Longissimus Thoracis & 39.02 & 43.50 & 70.25 & 36.69 & 43.93 & 46.68 \\
\hline
\end{tabular}

In the performance of Turkish folk dances, the left Tibialis Anterior muscle (112.40) and the left Quadriceps Femoris muscle (112.32) in the leg; the right Flexor Digitorum Superficialis muscle (49.63), the right Biceps Brachii (60.07) in the arm; the left Longissimus Thoracis muscle (46.68) and the right Trapezius muscle on the back were contracted more.

\section{CONCLUSION AND RECOMMENDATIONS}

In the performance of "bar" type dances;

- Left Tibialis Anterior and right Quadriceps Femoris muscles in the leg,

- Right Flexor Digitorum Superficialis and left Biceps Brachii muscles in the arm,

- The muscles of the right Longissimus Thoracis and left Trapezius on the back were contracted more.

In the performance of "Halay" type dances;

- Right Tibialis Anterior and Quadriceps Femoris muscles in the leg,

- Right Flexor Digitorum Superficialis and Biceps Brachii muscles in the arm,

- The left Longissimus Thoracis and the right Trapezius muscles on the back were contracted more.

In the performance of "Horon" type dances;

- Left Tibialis Anterior and Quadriceps Femoris muscles in the leg,

- Right Flexor Digitorum Superficialis and Biceps Brachii muscles in the arm,

- The muscles of the right Longissimus Thoracis and Trapezius on the back were contracted more.

In the performance of "karşılama" type dances;

- Right Tibialis Anterior and Left Quadriceps Femoris muscles in the leg,

- Right Flexor Digitorum Superficialis and Biceps Brachii muscles in the arm,

- The muscles of the right Longissimus Thoracis and left Trapezius on the back were contracted more.

In the performance of "Zeybek" type dances;

- Left Tibialis Anterior and Quadriceps Femoris muscles in the leg,

- Left Flexor Digitorum Superficialis and right Biceps Brachii muscles in the arm,

- The left Longissimus Thoracis and the right Trapezius muscles on the back were contracted more. 
Gülbeyaz, K. (2019). Comparison of activation rates in the main muscle group in the performance of Turkish folk dances. Journal of Human Sciences, 16(4), 1133-1144. doi:10.14687/jhs.v16i4.5888

On the basis of species;

- Left Tibialis Anterior and Quadriceps Femoris muscles in the leg,

- Right Flexor Digitorum Superficialis and Biceps Brachii muscles in the arm,

- The left Longissimus Thoracis and the right Trapezius muscles on the back were contracted more.

Folk dances include many basic motoric features such as flexibility, strength, coordination, skill and endurance. Therefore, it can be used in the development of basic motoric features of people of all ages, especially children of developmental age.

The desired muscle groups are developed with the determination and teaching of folk dances which use those muscle groups. In this way, folk dance can be used for training of other sports.

Since the whole body is used in a certain rhythmic structure in folk dances, it can be used in coordination (hand - arm - foot - body) exercises for athletes.

Folk dances can be used in physical therapy and rehabilitation with the determination of muscle utilization rates.

Folk dances can be used to lose weight. With the determination of the games that use the region to be given more weight, teaching and playing these games will result in a lot of work and weight loss.

Folk dances are a combination of regular and rhythmic movements. During the performance of dance, changes occur in all metabolism, especially in the respiratory and circulatory systems. These changes prove that folk dance is an important activity of physical activity. Therefore, in the area of folk dances, quantitative studies based on measurements, especially related to sport science, are very important. I believe that these kind of studies should be given importance, considering the fact that such studies are almost non-existent.

\section{REFERENCE}

Altuğ, N. (1988), "Music Related Problems in the Staging of Folk Dances" (Halk Oyunlanının Sahnelenmesinde Müzikle İlgili Sorunlar), Proceedings of Turkish Folk Dances Symposium, 26-28 October 1987, Middle East Technical University, Ministry of Culture and Tourism Publications Research Department Publications 102, Seminar, Congress Proceedings Series: 25, pp: 1-8, Öztek Printing House, Ankara.

Aydin, C. (1982), "Sportive Values of Folk Dances in Terms of Sociology" (Toplumbilim Açısından Halk Oyunlarının Sportif Değerleri), Ege University School of Physical Education Master Thesis, İzmir.

Ekmekçioğlu, İ., Bekar, C. ve Kaplan, M. (2001), “Turkish Folk Dances” (Türk Halk Oyunları), Esin Publishing, Istanbul.

Gülbeyaz, K. (2018), “Analysis of Turkish Folk Dances from the Perspective of Movement Science and Culture" (Hareket Bilimi ve Kültürel Açıdan Türk Halk Oyunlarının İncelenmesi), PhD Thesis, Sakarya University Institute of Social Sciences, Sakarya.

Huizinga, J. (1955), “An Essay on the Social Function of Homo Ludens's Dance” (Homo Ludens Oyunun Toplumsal İşlevi Üzerine Bir Deneme), Translated by Mehmet Ali, Kilıçbay, Detay Publications, İstanbul.

Kaya, Y. (2004), "Human Anatomy and Kinesiology" (İnsan Anatomisi ve Kinesyolojisi), Marmara Publishing, İstanbul. 

Journal of Human Sciences, 16(4), 1133-1144. doi:10.14687/ihs.v16i4.5888

Kaya, M. (2016), "Physical/Mental Dimensions of Turkish Folk Dance” (Türk Halk Oyunları Fiziksel/Zihinsel Boyutları), Product Publications, Ankara.

Öngel, H.B. (1992), “Origin of Turkish Folk Dances, Factors in Formation and Classification” (Türk Halk Oyunlarının Kökeni, Oluşumundaki Etkenler ve Sınıflandırılması), Gazi University, Institute of Health Sciences, Department of Physical Education and Sports, Master Thesis, Ankara.

Öter, Z. ve Ünal, E. (2017), “Contribution of Intangible Cultural Heritage and Tourism Interaction to Local Economy in Beypazarı District" (Somut Olmayan Kültürel Miras ve Turizm Etkileşiminin Beypazarı İlçesinde Yerel Ekonomiye Katkıları), 8th International Turkish Folk Culture Congress, 21-24 November 2011, Izmir, Ministry of Culture and Tourism Publications: 3519, Research and Education General Directorate: 378, C: 1, pp: 229-246, Ezgi Ofset Printing, Ankara.

Örnek, S.V. (2000), “Turkish Folklore” (Türk Halk Bilimi), Ministry of Culture Publication: 1629, 2nd Edition, Ankara.

Premkumar, K. (2015), “Anatomy and Physiology” (Anatomi ve Fizyoloji), LWW Massage Therapy and Exercise Training Series, Translation: Arzu Razak Özdinçler, Istanbul Medical Health and Publishing, Istanbul.

Weineck, J. (2011), "Fitness Training in Football" (Futbolda Kondisyon Antrenmanı), Translated by Tanju Bağırgan, Sports Publishing, Ankara. 